\title{
ATM-DEPENDENT CELLULAR RESPONSE TO DNA DOUBLE STRAND BREAKS PLAYS A PIVOTAL ROLE IN THE MAINTENANCE OF THE INTEGRITY OF THE GENOME
}

K. Suzuki*, M. Yamauchi and S. Yamashita Atomic Bomb Disease Institute, Graduate School of Biomedical Sciences, Nagasaki University, 1-12-4 Sakamoto, Nagasaki 852-8523, Japan

*Correspondence should be addressed to:

Keiji Suzuki, Ph.D.

kzsuzuki@nagasaki-u.ac.jp

Atomic Bomb Disease Institute, Graduate School of Biomedical Sciences, Nagasaki University, 1-12-4 Sakamoto, Nagasaki 852-8523, Japan 


\section{Abstract}

ATM-dependent cellular response to DNA double strand breaks plays a pivotal role in the maintenance of the integrity of the genome. Upon irradiation, Activated ATM proteins phosphorylate various downstream mediators and effectors, such as histone H2AX, MDC1, 53BP1 and NBS1. These proteins create discrete foci within the nuclei, which are detectable under fluorescence microscopes. Interestingly, the size of the foci is also increasing as increasing the time after irradiation. Particularly, the residual foci form large foci, whose sizes reach to approximately 2 micrometer in diameter. We confirmed that such "foci growth" is a mechanism, by which DNA damage signal is amplified. Especially, a proper DNA damage response of cells to lower doses of ionizing radiation required amplification of the ATM-dependent damage signal by recruiting the DNA damage checkpoint factors to the site of chromatin. 


\section{INTRODUCTION}

ATM-dependent cellular response to DNA double-strand breaks plays a pivotal role in maintaining genome integrity ${ }^{(1-6)}$. In response to ionizing radiation, autophosphorylation and monomerization of ATM proteins occur, and activated ATM phosphorylates various downstream mediators and effectors, such as histone H2AX, MDC1, 53BP1 and NBS1. These factors create discrete foci in the nuclei, which are detectable under fluorescence microscopy ${ }^{(7)}$. These foci are often called as ionizing radiation-induced foci (IRIF). The physiological importance of IRIF formation has been demonstrated by various studies, in which the cells lacking IRIF factors compromised proper DNA damage response, resulting in deficiencies in cell cycle arrest and DNA repair ${ }^{(1,2,5,6)}$.

Activated ATM mediates the phosphorylation of serine or threonine residues, which create specific docking sites for proteins harboring FHA and BRCT domains $^{(8)}$. In particular, phosphorylation of histone H2AX at serine 139 is the primary modification, which is essential for subsequent recruitment of IRIF factors $^{(9)}$. Furthermore, recruited proteins, including MDC1, NBS1, MRE11, and 53BP1, are also the targets for ATM-dependent phosphorylation, which is required for the sequential protein-to-protein interactions involved in IRIF formation $^{(10-15)}$. Thus, analyses of the dynamics of recruitment, phosphorylation, and IRIF formation are indispensable for a comprehensive understanding of DNA damage response to ionizing radiation.

Recently, we reported that the size of the IRIF foci drastically changed after irradiation $^{(16)}$. Particularly, residual foci, which persisted for over 24 hours after irradiation, showed more than $2 \mu \mathrm{m}$ in diameter. Since these "grown foci" were consistently detected in cells arrested at G1, it was highly likely that foci growth amplifies DNA damage signals. Moreover, the growth of the foci could be the indispensable mechanism for generating sufficient signals for executing G1 checkpoint in cells with a limited number of DNA damage. Thus, in the present 
study, we determined a molecular mechanism, by which foci size was changed. Also, the biological significance of DNA damage signal amplification was examined. 


\section{MATERIALS AND METHODS}

\section{Cell culture and $\mathrm{X}$-irradiation}

Normal human diploid fibroblast-like (NHD) cells were cultured in MEM supplemented with 10\% fetal bovine serum (FBS) (ThermoTrace Ltd, Australia). Chinese hamster ovary (CHO) and xrs5 cells were cultured in a-MEM supplemented with 10\% FBS. Cells were irradiated with various doses of X-rays from X-ray generator at $150 \mathrm{kVp}$ and $5 \mathrm{~mA}$ with a 0.1 -mm copper filter at a dose rate of $0.492 \mathrm{~Gy} / \mathrm{min}^{(16)}$. Synchronized cells were obtained by the mitotic shakeoff method, in which metaphase cells were collected by tapping the culture flasks. Most cells were in the G1 phase 6 hours after the mitotic shake-off.

\section{Immunofluorescence staining}

For immunofluorescence staining, cells were cultured on $22 \mathrm{~mm} \times 22 \mathrm{~mm}$ coverslips for 48 hours before irradiation. Cells were fixed with $4 \%$ formaldehyde, permeabilized with $0.5 \%$ Triton X-100, and then were washed extensively with phosphate buffered saline (PBS). The primary antibodies, antiphosphorylated histone H2AX monoclonal antibody (clone JBW, Upstate biotechnology, NY), anti-phosphorylated ATM monoclonal antibody (Rockland, PA), anti-MDC1 antibody (Bethyl laboratories Inc, TX), anti-NBS1 antibody (GeneTex, TX), anti-53BP1 antibody (Bethyl laboratories Inc, TX), and antiphosphorylated p53 antibody (Cell Signaling Technology, MA) were diluted in $100 \mathrm{ml}$ of TBS-DT (20 mM Tris-HCl, $137 \mathrm{mM} \mathrm{NaCl,} \mathrm{pH7.6,} \mathrm{containing} 50$ $\mathrm{mg} / \mathrm{ml}$ skim milk and $0.1 \%$ Tween-20), and applied on the cover slips. The samples were incubated for 2 hours in a humidified $\mathrm{CO}_{2}$ incubator at $37^{\circ} \mathrm{C}$. The primary antibodies were washed with PBS, and Alexa488- or Alexa594-labelled anti-mouse and anti-rabbit IgG antibodies (Molecular Probes, Inc., OR) were added, and the samples were incubated for 1 hour at $37^{\circ} \mathrm{C}$. After washing, they 
were counterstained with $0.1 \mathrm{mg} / \mathrm{ml}$ of 4',6-diamino-2-phenylindole (DAPI). The samples were examined with a F6000B fluorescence microscope (Leica, Tokyo). Digital images were captured and the images were analyzed by FW4000 software (Leica, Tokyo). In order to quantify the fluorescence intensity, maximum intensity projection images were used. The z-plane stacks of images of foci were captured at 0.2-0.3 mm intervals, and 15-20 images at each focal plane were collected. Then, regions at maximum intensity at each focal plane were assembled into 2D-images using FW4000. The green dot-like signals were marked on the screen, and the sum of the pixel intensity within the marked area was calculated as total fluorescence intensity.

\section{Cell cycle analysis}

In situ cell cycle distribution was examined using antibodies against replication protein A (RPA) and phosphorylated histone H3 at serine 10. Cells grown on 22 $\mathrm{mm}$ x $22 \mathrm{~mm}$ coverslips were treated with cytoskeleton stabilization buffer (10 mM HEPES, pH 7.4, $300 \mathrm{mM}$ Sucrose, $100 \mathrm{mM} \mathrm{NaCl}, 3 \mathrm{mM} \mathrm{MgCl}$ ) containing $0.5 \%$ Triton X-100 for 2 minutes on ice, fixed with $4 \%$ formaldehyde for 20 minutes in room temperature, permeabilized with $0.5 \%$ NP-40 for 5 minutes in room temperature, and then were washed extensively with phosphate buffered saline (PBS). The primary antibodies, anti-RPA antibody (Ab-3, Oncogene Research Product, MA), and anti-phosphorylated histone H3 at serine 10 antibody (Upstate Biotechnology Inc., NY) were diluted in $100 \mu \mathrm{l}$ of TBS-DT, and the samples were incubated with the antibodies for 2 hours in a humidified $\mathrm{CO}_{2}$ incubator at $37^{\circ} \mathrm{C}$. The primary antibodies were washed with $\mathrm{PBS}$, and Alexa488- or Alexa654-labelled anti-mouse and anti-rabbit IgG antibodies (Molecular Probes, Inc., OR) were added. The samples were incubated for 1 hour at $37^{\circ} \mathrm{C}$, and then washed with PBS and counterstained with $0.1 \mathrm{mg} / \mathrm{ml}$ of DAPI. The samples were examined with a fluorescence microscope as described. 


\section{RESULTS AND DISCUSSION}

\section{Foci growth after $\mathrm{X}$-irrdiation}

NHD cells were irradiated with 0.25 Gy of X-rays, and the foci of phosphorylated ATM were examined. Immediately after irradiation ( 1 min after irradiation), the initial detectable foci were tiny with a mean foci size of approximately 0.1 $\mu \mathrm{m}$. The foci size grew as the time after irradiation increased, with the mean size reaching a maximum at 30 minutes after irradiation. The number of foci, peaked at 30 minutes after $\mathrm{X}$-irradiation, decreased thereafter, and the average foci size also decreased concurrently. However, we found that some fractions of the initial foci were persisted depending on the dose. These foci, whose sizes were approximately $2.0 \mu \mathrm{m}$, were detectable even 1 week after irradiation. We found that such foci growth required the ATM kinase activity, as the inhibition of ATM activity by specific inhibitor compromised the foci growth. Thus, these results indicate that cells have evolved sophisticated mechanisms to amplify DNA damage signal by growing the foci size, and the foci growth occurs not simply by diffusion but depends on ATM activity.

It has been reported that phosphorylated histone H2AX foci and the foci of several DNA damage checkpoint factors are formed colocalized foci at the sites of chromatin where phosphorylated ATM foci are created ${ }^{(10-15)}$. We confirmed that all of the initial foci of phosphorylated histone H2AX, MDC1, 53BP1 and NBS1 were colocalized with phophorylated ATM foci. Interestingly, the sizes of these foci were increased as the size of phosphorylated ATM foci increased. Although the size of the persisted foci of 53BP1 was slightly larger than those of the other factors, the foci dynamics showed that all of them were necessary for the IRIF formation.

In response to ionizing radiation, ATM is activated through alteration of higher-order chromatin structure ${ }^{(17)}$. Therefore, the observed foci growth implied 
that the initial direct change in the chromatin structure was followed by the secondary disorganization of chromatin structure introduced by the subsequent biological process, i.e., DNA repair. To test this hypothesis, we compared cell cycle-dependent foci growth between $\mathrm{CHO}$ and xrs5 cells irradiated with $0.25 \mathrm{~Gy}$ of X-rays. Because xrs5 cells are lacking Ku80 protein, they are defective in nonhomologous end-joining (NHEJ), which is the major repair pathway for DNA double strand breaks in G1 phase. In fact, xrs5 cells in G1 phase are quite sensitive to radiation exposure. NHEJ is also involved in DNA repair in G2 cells, although the involvement is less significant. In the subsequent experiments, we analyzed the size of 53BP1 foci as they made more distinctive foci than phosphorylated ATM foci. We observed that both $\mathrm{CHO}$ and xrs5 cells showed the tiny initial foci 1 minute after irradiation in G1 and G2 phases (Figure 1). Thirty minutes after irradiation, the foci grew equally well in the G1 and G2 phases of CHO cells, however, in xrs5 cells, the foci growth was defective in the G1 phase cells (Figure 1). While the foci grew in the G2 phase, we also found that some foci were smaller than those observed in G2 CHO cells. These results indicated that the defective NHEJ in xrs5 cells affected the foci growth predominantly in the G1 phase. Thus, it can be concluded that the initial foci were formed through the initial chromatin structural change directly caused by DNA double strand breaks, however, the foci growth requires subsequent changes in the chromatin structure, which is provided by DNA repair.

Recently, it has been demonstrated that chromatin remodeling machineries are indispensable for DNA repair in chromatin in yeast and human cells ${ }^{(14-15,18)}$. However, the present observation indicates that DSB repair by itself causes subsequent dynamic change in the chromatin structure surrounding DSB, which results in the additional activation of DNA damage signaling. Therefore, DNA repair could be a necessary process that orchestrates chromatin remodeling for DNA ends-rejoining and amplification of DNA damage signal. The current idea 
was further supported by the evidences showing that the defect in NBS cells compromised both homologous recombination repair as well as DNA damage checkpoint $^{(1)}$.

\section{Foci growth and DNA damage signal amplification}

NHEJ-defective xrs5 cells failed to grow the initial DNA damage checkpoint foci in the G1 phase. Since ATM-dependent DNA damage signal is tightly related to the G1 checkpoint activation, defective foci growth possibly resulted in defective G1 checkpoint in xrs5 cells. As CHO and xrs5 cells have mutated p53 protein, it is impossible to check G1 arrest. However, the activation of G1 checkpoint is exclusively regulated by the activation of p53 function through ATM-dependent and site-specific phosphorylation ${ }^{(2)}$, therefore, we examined phosphorylation of p53 at serine 15 as a surrogate marker for p53 activation in G1-synchronized cells.

In response to $\mathrm{X}$-irradiation, immediate phosphorylation of p53 protein at serine 15 was visualized by anti-phosphorylated p53 at serine 15 antibody. The relative numbers of phosphorylated p53 molecules, as judged by the total fluorescence intensity, showed dose-dependent increase up to 4 Gy of X-rays in CHO cells (Figure 2). Similar dose-dependent activation of p53 was observed in xrs5 cells, however, the fluorescence intensity, especially at lower doses, was significantly compromised (Figure 2). For example, p53 activation at 1 Gy was less than $10 \%$ of that observed in CHO cells. At 2 Gy, p53 activation in xrs5 cells were about half of that in $\mathrm{CHO}$ cells, and no significant difference was observed with $4 \mathrm{~Gy}$ of X-rays. It was indicated that sufficient numbers of foci were induced at higher doses, which did not require amplification of DNA damage signal. Whereas, the foci number was significantly lower at lower doses, and each focus needed to be grown. Thus, while sufficient levels of DNA damage signal 
could be transmitted in xrs5 cells exposed to higher doses of radiation, G1 checkpoint might not be fully activated at lower doses in xrs5 cells.

As reported previously, the number of foci per Gy was different between NHD and CHO cells ${ }^{(19-21)}$. In NHD cells, the number of phosphorylated ATM foci was approximately 40 per Gy, while it was about 20 in CHO cells. Since dosedependent increase of foci number was linear up to $1 \mathrm{~Gy}$, it was expected that the numbers of foci per $10 \mathrm{mGy}$ were 0.4 and 0.2 per nuclei, respectively. Thus, theoretically, about half of NHD cells and one out of five CHO cells are estimated to have just one focus per cell. Although most of DNA double strand breaks induced by such low dose irradiation are reparable, it is essential to amplify DNA damage signal if DNA damage is left to be rejoined. It should be interesting to note that the number of molecules of DNA damage checkpoint factors per nucleus is limited. Therefore, if the number of the initial foci are higher, the foci growth may not be so apparent, while fewer foci can recruited more molecules per foci. In other words, this indicates that only a single focus is sufficient for DNA damage response if it can recruit most of the DNA damage checkpoint factors within the nucleus. Thus, amplification of the DNA damage signal through foci growth is one critical mechanism to maintain the integrity of the genome when cells were suffered by very low doses of radiation ${ }^{(16,21)}$. 


\section{CONCLUSION}

We demonstrated here that cells have a sophisticated mechanism, by which ATM-dependent DNA damage signal is amplified through the secondary change in the higher-order chromatin structure. This "foci growth" mechanism is coupled with DNA double strand break repair, and foci growth is a highly dynamic process involving several DNA damage checkpoint factors. As DNA damage is repaired, the foci becomes smaller and finally disappeared. However, if some fractions of the initial damage are persisted. they form large grown foci. Thus, the growth of the foci is an indispensable mechanism for generating sufficient signals for executing G1 checkpoint in cells with a limited number of DNA damage. With this, the integrity of the genome is securely protected from the detrimental effects of radiation. 


\section{ACKOWLEDGEMENTS}

This work was supported in part by the Nagasaki University Global Century Center of Excellence (COE) Program, and Grant-in-Aid for Scientific Research from the Ministry of Education, Culture, Sports, Science, and Technology, Japan 


\section{REFERENCES}

1. Shiloh, Y. ATM and related proteins kinases: safeguarding genome integrity. Nat. Rev. Cancer 3, 155-168 (2003).

2. Kitagawa, R. and Kastan, M.B. The ATM-dependent DNA damage signaling pathway. Cold Spring Harb. Symp. Quant. Biol. 70, 99-109 (2005).

3. Bartek, J., Bartkova, J. and Lukas, J. DNA damage signalling guards against activated oncogenes and tumour progression. Oncogene 26, 7773-7779 (2007).

4. Harper, J.W. and Elledge, S.J. The DNA damage response: ten years after. Mol. Cell 28, 739-745 (2007).

5. Lavin, M.F. Ataxia-telangiectasia: from a rare disorder to a paradigm for cell signalling and cancer. Nat. Rev. Mol. Cell. Biol. 9, 759-769 (2008).

6. Jackson S.P. and Bartek, L. The DNA-damage response in human biology and Disease. Nature 461, 1071-1078 (2009).

7. Paull, T.T., Rogakou, E.P., Yamazaki, V., Kirchgessner, C.U., Gellert, M. and Bonner, W.M. A critical role for histone H2AX in recruitment of repair factors to nuclear foci after DNA damage. Curr. Biol. 10, 886-895 (2000).

8. Matsuoka, S., Ballif, B.A., Smogorzewska, A., McDonald, E.R. 3rd., Hurov, K.E., Luo, J., Bakakarski, C.E., Zhao, Z., Solimini, N., Lerenthal, Y., Shiloh, Y., Gygi, S.P. and Elledge, S.J. ATM and ATR substrate analysis reveals extensive protein networks responsive to DNA damage. Science 316, 11601166 (2007).

9. Bonner, W.M., Redon, C.E., Dickey, J.S., Nakamura, A.J., Sedelnikova, O.A., Solier, S. and Pommier, Y. GammaH2AX and cancer. Nat. Rev. Cancer 8, 957-967 (2008).

10. Lukas, C., Bartek, J. and Lukas, J. Imaging of protein movement induced by chromosomal breakage: tiny 'local' lesions pose great 'global' challenges. Chromosoma 114, 146-154 (2005). 
11. Stucki, M. and Jackson, S.P. gammaH2AX and MDC1: anchoring the DNA-damage-response machinery to broken chromosomes. DNA Repair 5, 534-543 (2006).

12. Kinner, A., Wu, W., Staudt, C. and Iliakis, G. Gamma-H2AX in recognition and signalling of DNA double-strand breaks in the context of chromatin. Nucleic Acids Res. 36, 5678-5694 (2008).

13. FitzGerald, J.E., Grenon, M. and Lowndes, N.F. 53BP1: function and mechanism of focal recruitment. Biochem. Soc. Trans. 37, 897-904 (2009).

14. Misteli, T. and Soutoglou, E. The emerging role of nuclear architecture in DNA repair and genome maintenance. Nat. Rev. Mol. Cell Biol. 10, 243-254 (2009).

15. van Attikum, H. and Gasser, S.M. Crosstalk between modifications during the DNA damage response. Trends. Cell Biol. 19, 207-217 (2009).

16. Yamauchi, M., Oka, Y., Yamamoto, M., Niimura, K., Uchida, M., Kodama, S., Watanabe, M., Sekine, I., Yamashita, S. and Suzuki, K. Growth of persistent foci of DNA damage checkpoint factors is essential for amplification of G1 checkpoint signaling. DNA Repair 7, 405-417 (2008).

17. Bakkenist, C.J. and Kastan, M.B. DNA damage activates ATM through intermolecular autophosphorylation and dimer dissociation. Nature 421, 499506 (2003).

18. Altaf, M., Augar, A., Covic, M. and Cote, J. Connection between histone H2A variants and chromatin remodeling complexes. Biochem. Cell Biol. 87, 35-50 (2009).

19. Bekker-Jensen, S., Lukas, C., Kitagawa, R., Melander, F., Kastan, M.B., Bartek, J. and Lukas, J. Spatial organization of the mammalian genome surveillance machinery in response to DNA strand breaks. J. Cell Biol. 173, 195-206 (2006). 
20. Olive, P.L. Detection of DNA damage in individual cells by analysis of histone H2AX phosphorylation. Methods Cell Biol. 75, 355-373 (2004).

21. Suzuki, K., Okada, H., Yamauchi, M., Oka, Y., Kodama, S. and Watanabe, M. Qualitative and quantitative analysis of phosphorylated ATM foci induced by low dose ionizing radiation. Radiat. Res. 165, 499-504 (2006). 


\section{Figure legends}

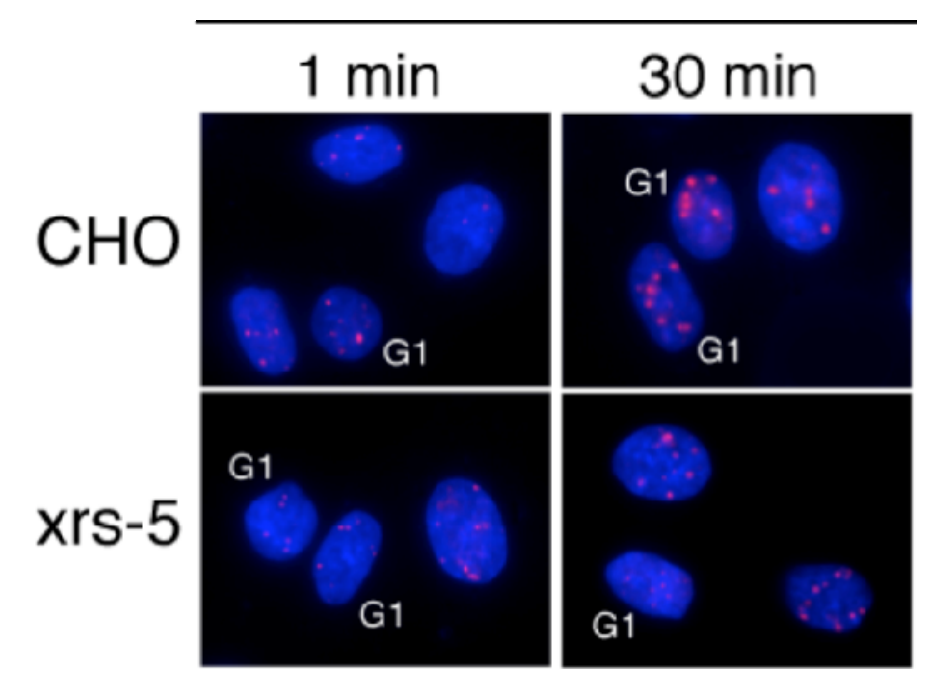

Figure 1 Cell cycle-dependent foci growth in $\mathrm{CHO}$ and xrs-5 cells

CHO and xrs-5 cells cultured on coverslips were irradiated with 0.25 Gy of Xrays, and growth of 53BP1 foci was examined 1 minute and 30 minutes after Xirradiation, as described in Materials and Methods. Cells in the S and G2 phases were identified by antibodies against RPA and phosphorylation of histone $\mathrm{H} 3$ at serine 10 , respectively. 

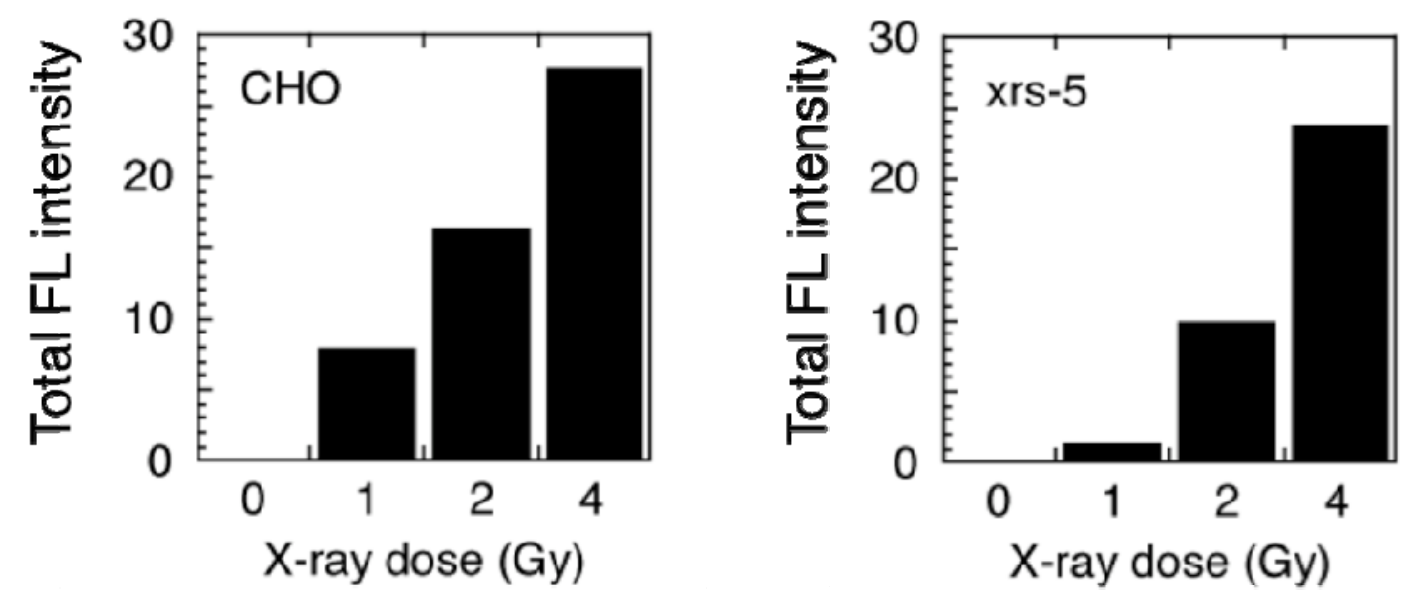

Figure 2 Phosphorylation of p53 in CHO and xrs-5 cells

G1-synchronized CHO and xrs-5 cells were obtained by the mitotic shake-off method, as described in Materials and Methods. They were cultured on coverslips and irradiated with various doses of X-rays. Phosphorylation of p53 at serine was examined 2 hours after $\mathrm{X}$-irradiation, as described in Materials and Methods. Total fluorescence intensity was calculated as the sum of the pixel intensity within the nuclear areas. 